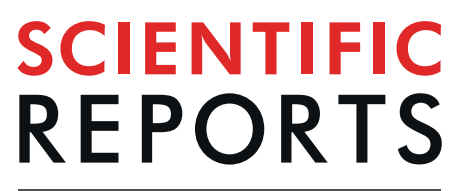

natureresearch

\title{
Effects of exergames on heart rate variability of women with fibromyalgia: A randomized controlled trial
}

\begin{abstract}
Santos Villafaina $\mathbb{1}^{1}$, Daniel Collado-Mateo ${ }^{2 *}$, Francisco J. Domínguez-Muñoz ${ }^{1}$, Narcis Gusi ${ }^{1}$ \& Juan P. Fuentes-Garcia ${ }^{3}$

The objective of the present manuscript was to evaluate the effects of 24-weeks exergame intervention on the heart rate variability (HRV) of women with fibromyalgia. First, 56 women with fibromyalgia were assessed for eligibility. A total of 55 women fulfilled the inclusion criteria and participated in this single-blinded, randomized controlled trial. A 24-weeks of exergames were completed by the exercise group in the university facilities. It was focused on the mobility, postural control, upper and lower limbs coordination, aerobic fitness and strength. A total of $120 \mathrm{~min}$ per week, divided into two sessions of $60 \mathrm{~min}$, was completed. A short-term $5 \mathrm{~min}$ record at rest was used to assess the HRV. Time (SDNN and RMSSD) and non-linear indexes (Higuchi's Fractal Dimension, SD1, SD2, In stress score, and SD1/ SD2) of HRV were extracted. Fifty participants (achieving an $\mathbf{8 9 . 2 8 \%}$ of adherence), recruited from the local fibromyalgia association completed the study. They were randomly divided into an exercise (age $=54.04[8.45]$ ) and a control group (52.72[9.98]). Significant interaction (group*time) effects in SDNN, In stress score, SD2, and SD1/SD2 ratio were found. The EG showed an increase of SDNN and a decreased In stress score and SD2. The CG showed an increased In stress score, SD1/SD2. In conclusion, 24-weeks of exergame intervention based on the tool VirtualEx-FM improved the autonomic control in patients with fibromyalgia. However, significant effects on Higuchi's fractal dimension were not found. This is the first study using exergame as a therapy in women with fibromyalgia which has led to an improvement the autonomic balance in these patients.
\end{abstract}

Fibromyalgia is a chronic syndrome characterized by widespread pain. It is frequently accompanied by other symptoms, such as fatigue, stiffness or sleep disturbance ${ }^{1}$. In addition, previous studies have suggested an autonomic nervous system dysfunction (dysautonomia) in patients with $\mathrm{FM}^{2-6}$. Dysautonomia is characterized by persistent autonomic nervous system hyperactivity at rest as well as hyporeactivity during stressful situations ${ }^{7}$. Therefore, the dysautonomia might explain some of the multisystem features of $\mathrm{FM}^{8}$.

The heart rate variability (HRV) (beat-to-beat variation in the R-R interval) is a reproducible and non-invasive measure of the autonomic nervous system function ${ }^{9}$. It provides information about the autonomic modulation (balance between the parasympathetic and the sympathetic nervous system) ${ }^{10}$. It is a relevant measure since low HRV values are associated with an increased risk of death from several causes ${ }^{11}$. In patients with FM, HRV has been used to assess the autonomic dysfunction ${ }^{2,3,5,6}$. Moreover, non-linear methods have been introduced in the study of HRV. Whereas in healthy circumstances it has been observed a presence of power-law fractal organization, a degradation of the fractal scaling has been observed in pathologic conditions ${ }^{12}$. In this regard, women with fibromyalgia exhibits decreased nonlinearity and stronger anticorrelations in heart period fluctuations ${ }^{13}$.

A systematic review with synthesis of best evidence pointed out that one of the non-pharmacological therapies with the highest level of evidence to manage fibromyalgia symptoms is physical exercise ${ }^{14}$. Physical exercise has shown to increase HRV in patients with fibromyalgia in aerobic exercise, resistance training or hydrotherapy interventions ${ }^{15-17}$. However, this may be controversial since some studies have reported no changes in HRV after exercise intervention programs ${ }^{18-20}$.

${ }^{1}$ Physical Activity and Quality of Life Research Group (AFYCAV). Faculty of Sport Science, University of Extremadura, Extremadura, Spain. ${ }^{2}$ Centre for Sport Studies, Rey Juan Carlos University, 28943, Fuenlabrada, Madrid, Spain. ${ }^{3}$ Faculty of Sport Science, University of Extremadura, Extremadura, Spain. *email: danicolladom@gmail.com 
Interventions based on virtual reality (VR) have emerged as a therapy in different populations $\mathrm{s}^{21-23}$. Exergames are a non-immersive variation of VR which involve physical exercise ${ }^{24}$. In this regard, a recent systematic review and meta-analysis indicated that musculoskeletal pain was reduced after exergame interventions, especially in patients suffering from chronic pain. In addition, in patients with fibromyalgia, an 8-week exergame intervention improved the mobility skills ${ }^{25}$ and the quality of life and pain ${ }^{26}$.

However, to the best of our knowledge, there is no study evaluating the effects of exergames on HRV in patients with fibromyalgia. Therefore, the aim of the present study was to evaluate the effects of 24-weeks exergame-based intervention on HRV in patients with fibromyalgia. Based on the previously cited scientific studies, we hypothesized that participants would increase their HRV after the exergame-based intervention. This would reduce the persistent autonomic nervous system hyperactivity in women with fibromyalgia.

\section{Method}

Trial design. This study was conceived as a single-blinded, randomized controlled trial. Participants were randomly allocated into two groups: exercise group (EG) and control group (CG). All the procedures were approved by the University of Extremadura research ethics committee (approval number: 62/2017) and performed in accordance with relevant guidelines and regulations. The trial was prospectively registered at the International Standard Randomised Controlled Trial Number Registry (ISRCTN65034180, date: 07/12/2017). The protocol is available on the following website: https://doi.org/10.1186/ISRCTN65034180.

However, some changes were accomplished. First, sample size was increased in order to obtain a greater statistical power. Second, the intervention was performed in the university facilities instead of in the local fibromyalgia association facilities.

Three articles focused on the primary outcomes of the trial have been recently published ${ }^{27-29}$. Nevertheless, the hypothesis in the present study is entirely novel (improvements in HRV after an exergame intervention) and significantly differs from the other articles. Furthermore, this article involves a different research, with specific research professionals and audience, enabling us to deeply examine the findings in the autonomic modulation of women with fibromyalgia.

Participants. The intervention was carried out in the University facilities (Faculty of Sport Science, Cáceres, Spain) from January 2018 to June 2018.

The fibromyalgia impact questionnaire was employed in order to estimate the sample size ${ }^{30}$. Taking into account that a $14 \%$ reduction is contemplated as clinically relevant ${ }^{31}$ and also that data from a previous research, the mean was expected to be $70.5(11.8)^{32}$. Therefore, 26 participants per group were estimated in order to detect differences ( $\alpha$ value 0.05 and $85 \%$ of power) of $14 \%$ in the fibromyalgia impact questionnaire.

Finally, a total of 56 women with fibromyalgia, from a fibromyalgia association were recruited until Dec 31, 2017. Participant inclusion criteria were:

(a) Be a woman between 30 and 75 years-old,

(b) Be able to communicate with the researchers and clinicians,

(c) Have read, accepted and signed the written informed consent,

(d) Be diagnosed with fibromyalgia by a rheumatologist according to the criteria of the American College of Rheumatology ${ }^{1}$.

In addition, participants were excluded if they:

(a) Modify their usual care therapies during the 24-week intervention,

(b) Have a condition that may make the physical exercise contraindicated according to a doctor, such as chronic or acute infectious diseases or renal, cardiac, pulmonary or hepatic failure.

(c) Be pregnant.

Participants were randomly allocated into the two groups (EG and CG) by one researcher using random numbers (EG and CG). The researcher who allocated the participants into the two groups did not take part in the acquisition or data analysis. A researcher who was blinded to the grouping allocation developed the evaluations. Nevertheless, participants were not blinded since they have to sign written informed consent about exergame intervention.

Interventions. EG consisted of 24-weeks of exergame-based intervention (two sessions of one hour per week) in groups of two or three participants. By contrast, the CG continued with their usual daily life. The intervention and evaluations were carried out in the university facilities. The intervention was delivered as planned.

The exercise intervention was based on the VirtualEx-FM exergame program. This is a tool specifically created by our research group which aimed to improve the ability to activities of daily living through the improvements in strength, postural control, aerobic fitness, mobility, and coordination of the upper and lower limbs ${ }^{26}$ in patients with fibromyalgia. Collado-Mateo, et al. ${ }^{26}$ and Collado-Mateo, et al. ${ }^{25}$ described in their study how this tool fulfilled with eight key points to consider the VirtualEx-FM as a virtual reality rehabilitation therapy ${ }^{33}$.

A typical session of the exergame intervention contained:

1. A warm-up where participants have to do joints movements guided by a video of a kinesiologist;

2. Aerobic component following the teacher's dance steps,

3. Coordination and postural control games where participants have to reach an apple that appears and disappears in different locations. The application indicated the body segment participants have to use. 
Moreover, it can be manually controlled by the kinesiologist; and,

4. Walking training where the participant must comprise a virtual trail of footprints. The interface allows the selection of different kind of steps such as: tiptoe, raised heels or knees, heel walking or normal. More details of the VirtualEx-FM are available in Collado-Mateo, et al. ${ }^{26}$ and Collado-Mateo, et al. ${ }^{25}$.

Outcomes. A short-term HRV register (5-minutes in sitting position) was recorded pre and post intervention. The heart rate monitor Polar RS800CX (Finland) was used ${ }^{34}$. Recommendations of the Task Force of the European Society of Cardiology and the North American Society of Pacing and Electrophysiology ${ }^{35}$ as well as instructions derived from previous studies were followed ${ }^{36,37}$. The Kubios HRV software (v. 2.1) . $^{38}$ was employed to extract time domain non-linear measures.

In order to correct artifacts, a medium filter was applied identifying all beat to beat intervals (RR) which were longer/shorter than 0.25 seconds (compared to the local average) ${ }^{39}$. A cubic spline interpolation replaced the artifacts. Low-frequency baseline trend components were removed using the smoothness prior method with a Lambda value of $500^{40}$.

Time-domain. SDNN: the standard deviation of all normal to normal RR intervals. Both sympathetic and parasympathetic systems contribute to SDNN. However, in short-term resting conditions the primary source of variations is parasympathetically-mediated by the respiratory sinus arrhythmia?

RMSSD: the root mean square of successive differences between RR intervals. Since this measure reflects the beat-to-beat variance, it is used to estimate the vagally mediated changes in $\mathrm{HRV}^{9,41}$.

Non-linear measures. SD1, SD2 and SD1/SD2 ratio: the standard deviation, of points perpendicular to the axis of line-of-identity in the Poincaré plot (SD1), the dispersion, standard deviation, of points along the axis of line-of-identity in the Poincaré plot (SD2) and the ratio between SD1 and SD2 (SD1/SD2 ratio) were calculated. The SD1 reflects the short-term HRV and it is identical to RMSSD measure ${ }^{42}$. The SD2 measures both, short and long-term HRV and correlates with $\mathrm{LF}^{43,44}$. The ratio SD1/SD2 is traditionally employed to measure the autonomic balance, correlating with the $\mathrm{LF} / \mathrm{HF}$ ratio $^{45,46}$.

ln stress score: the natural logarithm of the $1000 * 1 / \mathrm{SD} 2$. This index was created to provide a directly proportional value of the sympathetic activity ${ }^{47}$.

Higuchi Fractal dimension (HFD): HFD ${ }^{48}$ was calculated using the algorithm described by Khoa, et al. ${ }^{49}$ with the MATLAB R2016b software (The Mathworks Inc., Natick, MA, United States; Academic License, IBBE PAS). Firstly, as proposed by Kantelhardt, et al..$^{50}$ the original time series $y(\mathrm{i})$ were integrated from its mean, $\overline{\mathrm{y}}$, for every time point $i$, as follow:

$$
y(k)=\sum_{i=1}^{k}[y(i)-\bar{y}] \text { for } k=1, \ldots, N
$$

where $N$ is the length of the time series.

Subsequently, to obtain the fractal dimension, the N-length data series were split to a k-series set. This process calculates the length of the curve of each series in k-series set. The length for total curve was calculated using average. This gives $\mathrm{L}(\mathrm{k})$ for a $\mathrm{k}$ which varied from 1 to $\mathrm{Kmax}$. Kmax was calculated as: floor(length(data)/2). The fractal dimension was calculated over the entire 5-min epoch using all data points.

Statistical analysis. The SPSS statistical package (version 20.0; SPSS, Inc., Chicago, Ill.) was used to analyze the data.

Parametric tests were conducted based on the results of Shapiro-Wilk and Kolmogorov-Smirnov tests. Moreover, surrogate data were generated in order to test if the original data were derived from a stationary linear stochastic process with Gaussian inputs. This process consists of generating a surrogate data set with the same linear properties of the original RR data. If the comparison between surrogate data and RR time series is significantly different, the null hypothesis is rejected and nonlinearity assumed ${ }^{51,52}$. Surrogate data analysis was performed with the RHRV ${ }^{53}$, an open-source package for the statistical environment R (see: http://rhrv.r-forge.r-project.org/ index.html), following the steps of Martínez, et al. ${ }^{53}$. In addition, Chi-squared test was used to evaluate differences between control and exergame in the nonlinearity assumption.

Repeated Measures ANOVA with Bonferroni correction for multiple comparisons to avoid the increase of type I error was conducted to explore the effects of the exergame intervention. Within groups comparisons, between the pre and the post-tests, were performed by the T-tests for paired samples. Cohen's D effect size was reported for each statistical test ${ }^{54}$.

\section{Results}

The flow diagram of the participants is depicted in Fig. 1. A total of 56 patients with fibromyalgia were screened for eligibility. One woman did not meet the inclusion criteria, so she was excluded. Lastly, 55 women were randomized into two groups: EG and CG. Regarding the compliance with the treatment, 50 women finished the intervention program (EG: $\mathrm{n}=25$; age $=54.04$ [8.45] and CG: $\mathrm{n}=25$; age $=52.72$ [9.98]). Three women allocated in the EG and two in the CG were lost to follow-up. In the EG the causes were a surgery unrelated to the exercise intervention $(n=1)$ and the lack of time $(n=2)$. In the CG two women were not able to attend the final evaluations. The intervention was considered as completed when the participant attended a minimum of $75 \%$. Thus, considering this data the final adherence was $89.28 \%$. No side effects derived from the intervention were detected.

The main characteristics of the participants at baseline are summarized in Table 1. At baseline, no significant differences were reported in age nor the main outcomes (HRV indexes). 


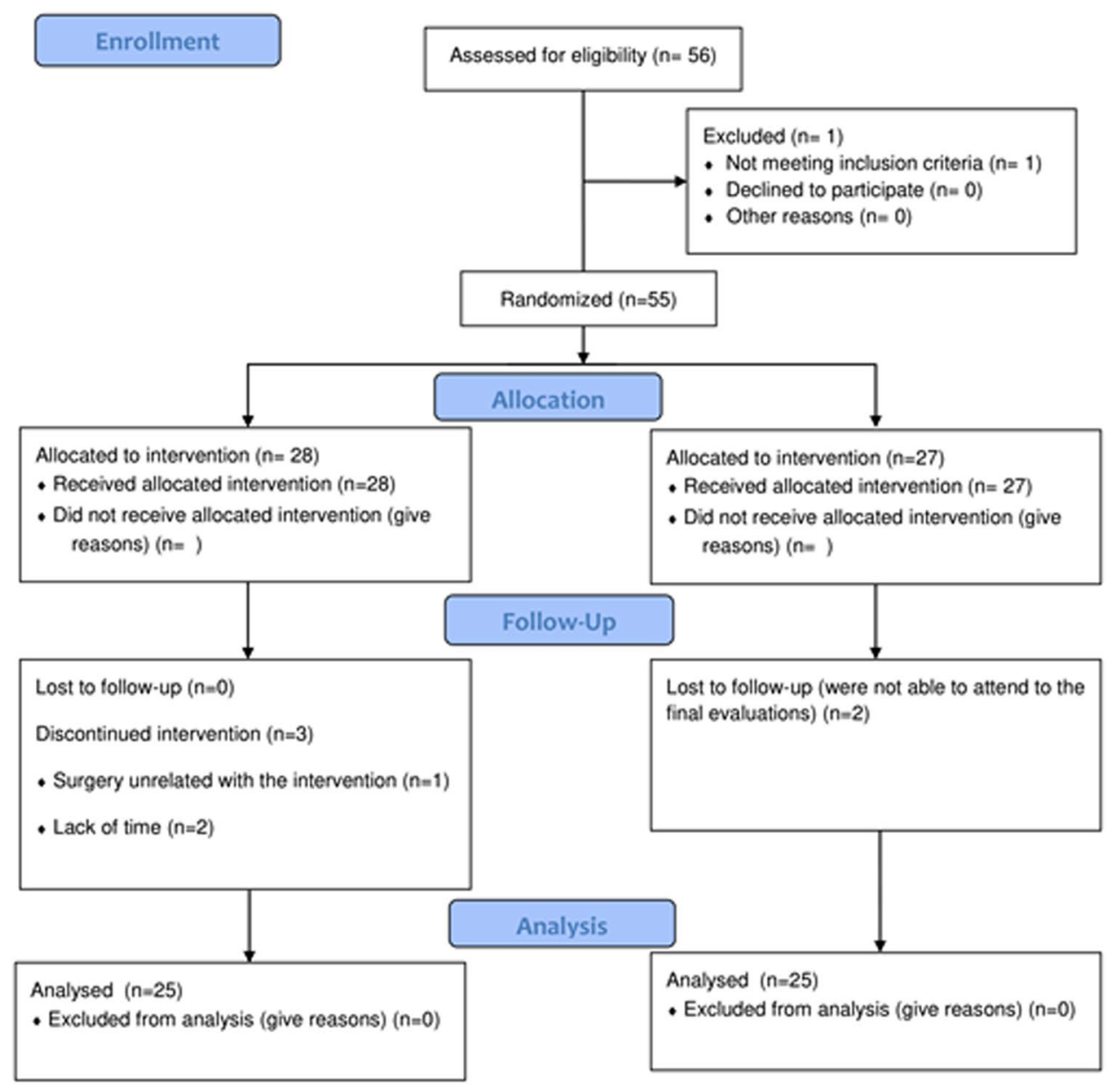

Figure 1. Flow diagram of the participants.

\begin{tabular}{|l|l|l|l|l|}
\hline Variable & Exercise Group Mean (SD) & Control Group Mean (SD) & Value of Contrast & p-value $^{\mathbf{a}}$ \\
\hline Sample size & 28 & 27 & & \\
\hline Age (years) & $54.04(9.96)$ & $53.41(9.92)$ & 0.234 & 0.816 \\
\hline SDNN $(\mathrm{ms})$ & $22.98(8.12)$ & $26.61(10.28)$ & -1.455 & 0.152 \\
\hline RMMSD $(\mathrm{ms})$ & $21.18(10.51)$ & $22.97(12.88)$ & -0.565 & 0.574 \\
\hline ln Stress Score & $3.61(0.33)$ & $3.47(0.44)$ & 1.261 & 0.213 \\
\hline SD1 (ms) & $15.01(7.44)$ & $16.26(9.12)$ & -0.561 & 0.577 \\
\hline SD2 (ms) & $28.55(9.49)$ & $33.59(12.39)$ & -1.697 & 0.096 \\
\hline SD1/SD2 & $0.52(0.15)$ & $0.47(0.16)$ & 1.251 & 0.216 \\
\hline HFD & $1.33(0.12)$ & $1.37(0.12)$ & -0.915 & 0.365 \\
\hline
\end{tabular}

Table 1. Demographic data and differences between groups at baseline. ${ }^{a} \mathrm{p}$-values of t-test for independent sample to compare differences between groups at baseline. SDNN: standard deviation of all normal to normal RR intervals; ln stress score: natural logarithm of the $1000 * 1 / \mathrm{SD} 2$; SD1: standard deviation of points perpendicular to the axis of line-of-identity in the Poincaré plot; SD2: standard deviation, of points along the axis of line-of-identity in the Poincare plot; SD1/SD2: ratio between SD1 and SD2; HFD: Higuchi Fractal Dimension.

Surrogate data was calculated for each participant in the pre and post RR time series. Nonlinearity, in the pre-test, is assumed in 24 participants of the exercise $(n=12,48 \%)$ and control $(n=12,48 \%)$ groups. Similar results were found in the post-test where nonlinearity is assumed in fourteen (32\%) and eleven (40\%) participants of the exergame and control group respectively. Chi-square test did not show differences between control and exergame groups in the nonlinearity assumption in both pre and post-tests (see Table 2). The efficacy analysis of the intervention is reported in Table 3. The repeated measures ANOVA showed significant interaction effects (time*group) in SDNN, ln stress score, SD2, and SD1/SD2 ratio. In this regard, in the EG, T-test for paired sample 


\begin{tabular}{|l|l|l|l|}
\hline \multicolumn{2}{|c|}{} & \multicolumn{2}{|l|}{ Non linearity assumed n (\%) } \\
\cline { 3 - 4 } \multicolumn{2}{|c|}{} & Pre & Post \\
\hline \multirow{2}{*}{ Surrogate data test } & Exercise & $12(48 \%)$ & $14(56 \%)$ \\
\cline { 2 - 4 } & Control & $12(48 \%)$ & $11(44 \%)$ \\
\hline p-value & 1.000 & 0.720 \\
\hline
\end{tabular}

Table 2. Surrogate data test analysis of the RR time series. ${ }^{\mathrm{a} p}$-values obtained from chi-square test.

\begin{tabular}{|c|c|c|c|c|c|c|c|c|c|}
\hline \multirow[b]{2}{*}{$\begin{array}{l}\text { Heart rate } \\
\text { variability } \\
\text { indexes }\end{array}$} & & \multirow[b]{2}{*}{ Pre } & \multirow[b]{2}{*}{ Post } & \multicolumn{3}{|c|}{ Within Group Comparison } & \multicolumn{3}{|c|}{ Between Group Comparison } \\
\hline & & & & $\begin{array}{l}\begin{array}{l}\text { Value } \\
\text { of the } \\
\text { contrast }\end{array} \\
\end{array}$ & P-value & $\begin{array}{l}\text { Effect } \\
\text { Size }\end{array}$ & F & P-value ${ }^{a}$ & $\begin{array}{l}\text { Effect } \\
\text { Size }\end{array}$ \\
\hline \multirow{2}{*}{ SDNN (ms) } & Exercise & $23.59(8.38)$ & $28.37(13.83)$ & -2.454 & 0.022 & -0.368 & \multirow{2}{*}{6.262} & \multirow{2}{*}{0.016} & \multirow{2}{*}{0.721} \\
\hline & Control & $25.64(10.06)$ & $20.97(12.28)$ & 1.443 & \begin{tabular}{|l|}
0.162 \\
\end{tabular} & 0.415 & & & \\
\hline \multirow{2}{*}{ RMSSD (ms) } & Exercise & $21.56(11.06)$ & $24.28(16.97)$ & -0.806 & 0.428 & -0.333 & \multirow{2}{*}{0.592} & \multirow{2}{*}{0.446} & \multirow{2}{*}{0.220} \\
\hline & Control & $21.07(11.11)$ & $20.14(14.36)$ & 0.280 & 0.782 & 0.073 & & & \\
\hline \multirow{2}{*}{ In Stress Score } & Exercise & $3.58(0.33)$ & $3.42(0.42)$ & 3.119 & 0.005 & 0.414 & \multirow{2}{*}{7.739} & \multirow{2}{*}{0.008} & \multirow{2}{*}{0.804} \\
\hline & Control & $3.50(0.45)$ & $3.83(0.66)$ & -1.950 & 0.063 & -0.587 & & & \\
\hline \multirow{2}{*}{$\mathrm{SD} 1$ (ms) } & Exercise & $15.27(7.83)$ & $17.20(12.01)$ & -0.807 & 0.428 & -0.187 & \multirow{2}{*}{0.593} & \multirow{2}{*}{0.445} & \multirow{2}{*}{0.220} \\
\hline & Control & $14.92(7.87)$ & $14.26(10.17)$ & 0.279 & 0.783 & 0.072 & & & \\
\hline \multirow{2}{*}{$\mathrm{SD} 2$ (ms) } & Exercise & $29.37(9.73)$ & $35.86(16.36)$ & -3.124 & 0.005 & -0.395 & \multirow{2}{*}{9.024} & \multirow{2}{*}{0.004} & \multirow{2}{*}{0.866} \\
\hline & Control & $32.82(12.55)$ & $25.77(14.49)$ & 1.763 & 0.091 & 0.520 & & & \\
\hline \multirow{2}{*}{$\mathrm{SD} 1 / \mathrm{SD} 2$} & Exercise & $0.52(0.16)$ & $0.46(0.17)$ & 1.224 & 0.233 & 0.351 & \multirow{2}{*}{6.897} & \multirow{2}{*}{0.012} & \multirow{2}{*}{0.759} \\
\hline & Control & $0.44(0.13)$ & $0.54(0.14)$ & -2.748 & 0.011 & -0.687 & & & \\
\hline \multirow{2}{*}{ HFD } & Exercise & $1.33(0.12)$ & $1.34(0.15)$ & -0.132 & 0.896 & -0.034 & \multirow{2}{*}{1.010} & \multirow{2}{*}{0.320} & \multirow{2}{*}{0.293} \\
\hline & Control & $1.37(0.12)$ & $1.32(0.11)$ & 1.250 & 0.224 & -0.320 & & & \\
\hline
\end{tabular}

Table 3. Efficacy analysis of exergame intervention in the HRV indexes in patients with fibromyalgia.

showed an increase of SDNN and a decrease of ln stress score and SD2. In the CG, T-test for paired sample showed an increase of ln stress score (see Table 3). No significant effects were obtained in the remaining HRV indexes (see Table 3).

\section{Discussion}

The aim of the present study was to evaluate the effects of a 24-weeks exergame intervention on the HRV in patients with fibromyalgia. Exergames have been used previously to improve both physical function and quality of life in women with fibromyalgia after 8 -week ${ }^{25,26}$ and 24 -week ${ }^{27,28}$ interventions. However, this is, to our knowledge, the first study to report a significant effect of an exergame-based intervention on the HRV of patients with fibromyalgia.

Fibromyalgia patients showed an abnormal autonomic modulation compared with healthy controls ${ }^{6,7}$. Importantly, our results showed that exergame promoted a change in autonomic modulation after the intervention by a significant increase in SDNN and SD2 and a significant decrease in ln Stress Score and SD1/SD2. Similar results have been observed in previous studies. In this regard, in a 24-weeks of aerobic training, fibromyalgia patients improved HRV measures such as $\operatorname{lnLF}$ and $\operatorname{lnLF} / \mathrm{HF}^{15}$. In the same line, Zamuner, et al. ${ }^{17}$ found that 16-weeks of hydrotherapy intervention (based on aerobic, resistance and stretching exercises) improved the HRV when compared with healthy controls (lnHF measure). Furthermore, 16-weeks of resistance training ${ }^{16}$ showed changes in the HRV (total power, lnHF and RMSSD measures), when compared with healthy controls. Similar effects were found after 12 -week Tai Chi intervention (significant effects in $\operatorname{lnLF} / \mathrm{HF}$ ratio, $\operatorname{lnHF}$ and $\operatorname{lnLF})^{55}$. However, there are also previous studies that did not find significant changes on HRV ${ }^{18-20}$ after 16 weeks of strengthening ${ }^{18}, 8$ weeks of resistance exercise ${ }^{19}, 12$ weeks of resistance training respectively ${ }^{20}$ and 12 weeks of moderate intensity spinning workouts ${ }^{56}$. Our results support the idea of vagal tone recovery due to exercise interventions. Furthermore, considering both our results and the previous research, it can be hypothesized that interventions longer than 4 months and comprising resistance or aerobic exercises are necessary to improve HRV. This hypothesis is also supported by previous studies in other populations ${ }^{57,58}$.

The non-linear measures seem to be more sensitive to autonomic modulation changes induced by exercise intervention than time measures since significant effects were found in the ln stress score, SD2 and the SD1/SD2 ratio. One of these indices, the ln stress score, was originally developed to control the autonomic balance in elite soccer players ${ }^{47}$. However, our results indicate that this index could be an interesting tool to measure the sympathetic activity, also in a different population with abnormal autonomic modulation such as fibromyalgia. This index is also derived from the Poincaré plot, i.e. $\ln$ stress score $=\ln \left(1000^{*} 1 / \mathrm{SD} 2\right)$. However, significant effects of exergames in the chaotic behavior of HRV measured by the HFD have been not found. The HFD is an appropriate method for analyzing the fractal dimension of biomedical signals ${ }^{59}$ in order to quantify self-similarity and complexity of the signal, providing more accurate estimation of fractal dimension than other proposed methods ${ }^{59,60}$. 
It has been applied to brain ${ }^{61}$ cardiac $^{62}$ or even center of pressure signals ${ }^{63}$. In this regard, a previous study with healthy and diabetic populations used the HFD to determine the effects of Percutaneous Auricular Vagus Nerve Stimulation on the Autonomic Nervous System ${ }^{64}$. Moreover, a decrease in fractal dimension has been observed under stress situations ${ }^{65}$. This is in line with the acute decrease of HFD after physical exercise ${ }^{66}$. However, further studies are needed to completely understand the interrelation of HFD with Autonomic Nervous System measures.

These results are relevant since lower values of HRV are related to high risk of mortality ${ }^{11}$. In addition, a previous study reported that improvements in HRV non-linear indexes were related to improvement in pain, quality of life and impact of the disease ${ }^{17}$. In this regard, Martinez-Lavin, et al. ${ }^{67}$ proposed that the sympathetic hyperactivity could be related to insomnia, anxiety and chronic pain suffered by patients with fibromyalgia. This could be explained by hyperactivity of the sympathetic nervous systems to stress, resulting of the down-regulation and desensitization of adrenergic receptors.

Nonlinear dynamics are involved in the creation of HRV due to different hemodynamic electrophysiological and autonomic/central regulations ${ }^{68,69}$. In this regard, surrogate data analysis has emerged as one of the most popular test to determine the presence of nonlinear dynamics in the RR time series ${ }^{69}$, assessing the capability of the different nonlinear indexes to detect nonlinearity in HRV data ${ }^{70-73}$. Our results showed that nonlinearity is assumed in $49 \%$ of the total RR data series (including pre and post-tests). Therefore, results from non-linear variables, such as HFD, In Stress Score, SD1, SD2 and SD1/SD2, must be taken with caution. Nevertheless, differences between control and exergame groups in the nonlinearity assumption were not found.

Study limitations. This study has some limitations that should be mentioned. First, a third group that performed exercise training intervention not based in VR should be recommended to isolate the effects of an exergame intervention. This fact makes that the effects of VR intervention should be taken with caution. Second, due to the relatively small sample probably only great differences have reached the statistical significance level. Third, in the present study only participated women, so we cannot generalize the results to men with fibromyalgia. Fourth, regarding non-linear analysis surrogate data analysis indicated that in $49 \%$ of RR data series intervals nonlinearity is assumed. Therefore, results from non-linear variables should be taken with caution.

\section{Conclusions}

To summarize, 24-weeks of exergame intervention based on the tool VirtualEx-FM improved the autonomic control in patients with fibromyalgia. However, effects of exergames chaotic behavior of HRV measured through the HFD were not found. This is the first study using exergames as a therapy in women with fibromyalgia achieving improvements in the autonomic control.

Received: 30 August 2019; Accepted: 24 February 2020;

Published online: 20 March 2020

\section{References}

1. Wolfe, F. et al. The American College of Rheumatology Preliminary Diagnostic Criteria for Fibromyalgia and Measurement of Symptom Severity. Arthrit Care Res 62, 600-610, https://doi.org/10.1002/acr.20140 (2010).

2. Raj, S. R., Brouillard, D., Simpson, C. S., Hopman, W. M. \& Abdollah, H. Dysautonomia among patients with fibromyalgia: a noninvasive assessment. The Journal of rheumatology 27, 2660-2665 (2000).

3. Solano, C. et al. Autonomic dysfunction in fibromyalgia assessed by the Composite Autonomic Symptoms Scale (COMPASS). Journal of clinical rheumatology: practical reports on rheumatic \& musculoskeletal diseases 15, 172-176, https://doi.org/10.1097/ RHU.0b013e3181a1083d (2009).

4. Eisinger, J. D. fibromyalgia and reflex dystrophy. Arthritis research \& therapy 9, 105, https://doi.org/10.1186/ar2212 (2007).

5. Ulas, U. H. et al. Dysautonomia in fibromyalgia syndrome: sympathetic skin responses and RR interval analysis. Rheumatology international 26, 383-387, https://doi.org/10.1007/s00296-005-0007-1 (2006).

6. Martinez-Lavin, M. Fibromyalgia as a sympathetically maintained pain syndrome. Current pain and headache reports $\mathbf{8}, 385-389$ (2004).

7. Furlan, R. et al. Abnormalities of cardiovascular neural control and reduced orthostatic tolerance in patients with primary fibromyalgia. The Journal of rheumatology 32, 1787-1793 (2005).

8. Martinez-Lavin, M. \& Hermosillo, A. G. Autonomic nervous system dysfunction may explain the multisystem features of fibromyalgia. Seminars in arthritis and rheumatism 29, 197-199, https://doi.org/10.1016/s0049-0172(00)80008-6 (2000).

9. Shaffer, F. \& Ginsberg, J. P. An Overview of Heart Rate Variability Metrics and Norms. Frontiers in public health 5, 258, https://doi. org/10.3389/fpubh.2017.00258 (2017).

10. Freeman, J. V., Dewey, F. E., Hadley, D. M., Myers, J. \& Froelicher, V. F. Autonomic nervous system interaction with the cardiovascular system during exercise. Progress in Cardiovascular Diseases 48, 342-362, https://doi.org/10.1016/j.pcad.2005.11.003 (2006).

11. Dekker, J. M. et al. Low heart rate variability in a 2-minute rhythm strip predicts risk of coronary heart disease and mortality from several causes - The ARIC study. Circulation 102, 1239-1244 (2000).

12. Guzman-Vargas, L. \& Angulo-Brown, F. Simple model of the aging effect in heart interbeat time series. Physical review. E, Statistical, nonlinear, and soft matter physics 67, 052901, https://doi.org/10.1103/PhysRevE.67.052901 (2003).

13. Reyes-Manzano, C. F. et al. Multifractal Analysis Reveals Decreased Non-linearity and Stronger Anticorrelations in Heart Period Fluctuations of Fibromyalgia Patients. Frontiers in physiology 9, 1118, https://doi.org/10.3389/fphys.2018.01118 (2018).

14. Bidonde, J., Jean Busch, A., Bath, B. \& Milosavljevic, S. Exercise for adults with fibromyalgia: an umbrella systematic review with synthesis of best evidence. Current rheumatology reviews 10, 45-79 (2014).

15. Sanudo, B., Carrasco, L., de Hoyo, M., Figueroa, A. \& Saxton, J. M. Vagal modulation and symptomatology following a 6-month aerobic exercise program for women with fibromyalgia. Clin Exp Rheumatol 33, S41-45 (2015).

16. Figueroa, A., Kingsley, J. D., McMillan, V. \& Panton, L. B. Resistance exercise training improves heart rate variability in women with fibromyalgia. Clinical physiology and functional imaging 28, 49-54, https://doi.org/10.1111/j.1475-097X.2007.00776.x (2008).

17. Zamuner, A. R. et al. Effects of a hydrotherapy programme on symbolic and complexity dynamics of heart rate variability and aerobic capacity in fibromyalgia patients. Clin Exp Rheumatol 33, S73-81 (2015).

18. Glasgow, A., Stone, T. M. \& Kingsley, J. D. Resistance Exercise Training on Disease Impact, Pain Catastrophizing and Autonomic Modulation in Women with Fibromyalgia. International journal of exercise science 10, 1184-1195 (2017). 
19. Kingsley, J. D., McMillan, V. \& Figueroa, A. The effects of 12 weeks of resistance exercise training on disease severity and autonomic modulation at rest and after acute leg resistance exercise in women with fibromyalgia. Archives of physical medicine and rehabilitation 91, 1551-1557, https://doi.org/10.1016/j.apmr.2010.07.003 (2010).

20. Gavi, M. B. et al. Strengthening exercises improve symptoms and quality of life but do not change autonomic modulation in fibromyalgia: a randomized clinical trial. PloS one 9, e90767, https://doi.org/10.1371/journal.pone.0090767 (2014).

21. Adamovich, S. V., Fluet, G. G., Tunik, E. \& Merians, A. S. Sensorimotor training in virtual reality: a review. NeuroRehabilitation 25, 29-44, https://doi.org/10.3233/nre-2009-0497 (2009).

22. Jansen-Kosterink, S. M. et al. A Serious Exergame for Patients Suffering from Chronic Musculoskeletal Back and Neck Pain: A Pilot Study. Games for health journal 2, 299-307, https://doi.org/10.1089/g4h.2013.0043 (2013).

23. Park, E. C., Kim, S. G. \& Lee, C. W. The effects of virtual reality game exercise on balance and gait of the elderly. Journal of physical therapy science 27, 1157-1159, https://doi.org/10.1589/jpts.27.1157 (2015).

24. Wuest, S., van de Langenberg, R. \& de Bruin, E. D. Design considerations for a theory-driven exergame-based rehabilitation program to improve walking of persons with stroke. European review of aging and physical activity: official journal of the European Group for Research into Elderly and Physical Activity 11, 119-129, https://doi.org/10.1007/s11556-013-0136-6 (2014).

25. Collado-Mateo, D., Dominguez-Muñoz, F. J., Adsuar, J. C., Merellano-Navarro, E. \& Gusi, N. Exergames for women with fibromyalgia: a randomised controlled trial to evaluate the effects on mobility skills, balance and fear of falling. PeerJ 5, e3211 (2017).

26. Collado-Mateo, D., Dominguez-Munoz, F. J., Adsuar, J. C., Garcia-Gordillo, M. A. \& Gusi, N. Effects of Exergames on Quality of Life, Pain, and Disease Effect in Women With Fibromyalgia: A Randomized Controlled Trial. Archives of physical medicine and rehabilitation 98, 1725-1731, https://doi.org/10.1016/j.apmr.2017.02.011 (2017).

27. Martín-Martínez, J. P., Villafaina, S., Collado-Mateo, D., Perez-Gomez, J. \& Gusi, N. Effects of 24-wk exergame intervention on physical function under single- and dual-task conditions in fibromyalgia. Scandinavian Journal of Medicine and Science in Sports (2019).

28. Villafaina, S., Collado-Mateo, D., Domínguez-Muñoz, F. J., Fuentes, J. P. \& Gusi, N. Benefits of 24-week exergames intervention on health-related quality of life and pain in women with fibromyalgia: a single-blind, randomized, controlled trial. Games for Health Journal (2019).

29. Villafaina, S., Collado-Mateo, D., Fuentes, J. P., Rohlfs-Dominguez, P. \& Gusi, N. Effects of Exergames on Brain Dynamics in Women with Fibromyalgia: A Randomized Controlled Trial. Journal of clinical medicine 8, https://doi.org/10.3390/jcm8071015 (2019).

30. Bennett, R. The Fibromyalgia Impact Questionnaire (FIQ): a review of its development, current version, operating characteristics and uses. Clin Exp Rheumatol 23, S154-162 (2005).

31. Bennett, R. M., Bushmakin, A. G., Cappelleri, J. C., Zlateva, G. \& Sadosky, A. B. Minimal clinically important difference in the fibromyalgia impact questionnaire. The Journal of rheumatology 36, 1304-1311, https://doi.org/10.3899/jrheum.081090 (2009).

32. Esteve-Vives, J., Rivera Redondo, J. \& Isabel Salvat Salvat, M. de Gracia Blanco, M. \& de Miquel, C. A. [Proposal for a consensus version of the Fibromyalgia Impact Questionnaire (FIQ) for the Spanish population]. Reumatologia clinica 3, 21-24, https://doi. org/10.1016/s1699-258x(07)73594-5 (2007).

33. Lewis, G. N. \& Rosie, J. A. Virtual reality games for movement rehabilitation in neurological conditions: how do we meet the needs and expectations of the users? Disability and rehabilitation 34, 1880-1886, https://doi.org/10.3109/09638288.2012.670036 (2012).

34. da Costa, M. P., da Silva, N. T., de Azevedo, F. M., Pastre, C. M. \& Marques Vanderlei, L. C. Comparison of Polar((R)) RS800G3 heart rate monitor with Polar((R)) S810i and electrocardiogram to obtain the series of RR intervals and analysis of heart rate variability at rest. Clinical Physiology and Functional Imaging 36, 112-117, https://doi.org/10.1111/cpf.12203 (2016).

35. Camm, A. J. et al. Heart rate variability. Standards of measurement, physiological interpretation, and clinical use. European heart journal 17, 354-381 (1996).

36. Sánchez-Molina, J., Robles-Pérez, J. J. \& Clemente-Suárez, V. J. Assessment of psychophysiological response and specific fine motor skills in combat units. Journal of medical systems 42, 67 (2018).

37. Beltrán-Velasco, A. I., Bellido-Esteban, A., Ruisoto-Palomera, P. \& Clemente-Suárez, V. J. Use of Portable Digital Devices to Analyze Autonomic Stress Response in Psychology Objective Structured Clinical Examination. Journal of medical systems 42, 35 (2018).

38. Tarvainen, M. P., Niskanen, J.-P., Lipponen, J. A., Ranta-aho, P. O. \& Karjalainen, P. A. Kubios HRV - Heart rate variability analysis software. Computer Methods and Programs in Biomedicine 113, 210-220, https://doi.org/10.1016/j.cmpb.2013.07.024 (2014).

39. Aranda, C., de la Cruz Torres, B. \& Orellana, J. N. Effects of different automatic filters on the analysis of heart rate variability with Kubios HRV software. Archivos de medicina del deporte: revista de la Federación Española de Medicina del Deporte y de la Confederación Iberoamericana de Medicina del Deporte, 196-200 (2017).

40. Tarvainen, M. P. \& Ranta-Aho, P. O. \& Karjalainen, P. A. J. I. T. o. B. E. An advanced detrending method with application to HRV. analysis. 49, 172-175 (2002).

41. Shaffer, F., McCraty, R. \& Zerr, C. L. A healthy heart is not a metronome: an integrative review of the heart's anatomy and heart rate variability. Frontiers in psychology 5, 1040-1040, https://doi.org/10.3389/fpsyg.2014.01040 (2014).

42. Ciccone, A. B. et al. Reminder: RMSSD and SD1 are identical heart rate variability metrics. Muscle \& nerve 56, 674-678, https://doi. org $/ 10.1002 /$ mus.25573 (2017).

43. Brennan, M., Palaniswami, M. \& Kamen, P. Do existing measures of Poincare plot geometry reflect nonlinear features of heart rate variability? IEEE transactions on bio-medical engineering 48, 1342-1347, https://doi.org/10.1109/10.959330 (2001).

44. Tulppo, M. P., Makikallio, T. H., Takala, T. E., Seppanen, T. \& Huikuri, H. V. Quantitative beat-to-beat analysis of heart rate dynamics during exercise. The American journal of physiology 271, H244-252, https://doi.org/10.1152/ajpheart.1996.271.1.H244 (1996).

45. Behbahani, S., Dabanloo, N. J. \& Nasrabadi, A. M. Ictal Heart Rate Variability Assessment with Focus on Secondary Generalized and Complex Partial Epileptic Seizures. Advances in Bioresearch 4 (2013).

46. Guzik, P. et al. Correlations between Poincaré plot and conventional heart rate variability parameters assessed during paced breathing. The Journal of Physiological Sciences, 0702020009-0702020009 (2007).

47. Naranjo Orellana, J., de la Cruz Torres, B., Sarabia Cachadina, E., de Hoyo, M. \& Dominguez Cobo, S. Two new indexes for the assessment of autonomic balance in elite soccer players. International journal of sports physiology and performance 10, 452-457, https://doi.org/10.1123/ijspp.2014-0235 (2015).

48. Higuchi, T. Approach to an irregular time series on the basis of the fractal theory. Physica D: Nonlinear Phenomena 31, 277-283 (1988).

49. Khoa, T. Q. D., Ha, V. Q. \& Toi, V. V. Higuchi fractal properties of onset epilepsy electroencephalogram. Computational and mathematical methods in medicine 2012 (2012).

50. Kantelhardt, J. W. et al. Multifractal detrended fluctuation analysis of nonstationary time series. Physica A: Statistical Mechanics and its Applications 316, 87-114 (2002).

51. Schreiber, T. \& Schmitz, A. Surrogate time series. Physica D: Nonlinear Phenomena 142, 346-382 (2000)

52. Theiler, J., Eubank, S., Longtin, A., Galdrikian, B. \& Farmer, J. D. Testing for nonlinearity in time series: the method of surrogate data. Physica D: Nonlinear Phenomena 58, 77-94 (1992).

53. Martínez, C. A. G. et al. Heart rate variability analysis with the R package RHRV. (Springer, 2017).

54. Fritz, C. O., Morris, P. E. \& Richler, J. J. Effect size estimates: current use, calculations, and interpretation. Journal of experimental psychology: General 141, 2 (2012).

55. Wong, A. et al. Effectiveness of Tai Chi on Cardiac Autonomic Function and Symptomatology in Women With Fibromyalgia: A Randomized Controlled Trial. Journal of aging and physical activity 26, 214-221, https://doi.org/10.1123/japa.2017-0038 (2018).

56. Bardal, E. M., Roeleveld, K. \& Mork, P. J. Aerobic and cardiovascular autonomic adaptations to moderate intensity endurance exercise in patients with fibromyalgia. Journal of rehabilitation medicine 47, 639-646, https://doi.org/10.2340/16501977-1966 (2015). 
57. Villafaina, S., Collado-Mateo, D., Fuentes, J. P., Merellano-Navarro, E. \& Gusi, N. Physical Exercise Improves Heart Rate Variability in Patients with Type 2 Diabetes: A Systematic Review. Current diabetes reports 17, 110, https://doi.org/10.1007/s11892-017-0941-9 (2017).

58. Hottenrott, K., Hoos, O. \& Esperer, H. D. [Heart rate variability and physical exercise. Current status]. Herz 31, 544-552, https://doi. org/10.1007/s00059-006-2855-1 (2006).

59. Accardo, A., Affinito, M., Carrozzi, M. \& Bouquet, F. Use of the fractal dimension for the analysis of electroencephalographic time series. Biological cybernetics 77, 339-350 (1997).

60. Esteller, R., Vachtsevanos, G., Echauz, J. \& Litt, B. A comparison of waveform fractal dimension algorithms. IEEE Transactions on Circuits and Systems I: Fundamental Theory and Applications 48, 177-183 (2001).

61. Ferenets, R. et al. Comparison of entropy and complexity measures for the assessment of depth of sedation. IEEE Transactions on Biomedical Engineering 53, 1067-1077 (2006).

62. Acharya, R., Bhat, P. S., Kannathal, N., Rao, A. \& Lim, C. M. Analysis of cardiac health using fractal dimension and wavelet transformation. ITBM-RBM 26, 133-139 (2005).

63. Doyle, T. L. A., Dugan, E. L., Humphries, B. \& Newton, R. U. Discriminating between elderly and young using a fractal dimension analysis of centre of pressure. International journal of medical sciences 1, 11 (2004).

64. Gomolka, R. S. et al. Higuchi fractal dimension of heart rate variability during percutaneous auricular vagus nerve stimulation in healthy and diabetic subjects. Frontiers in physiology 9, 1162 (2018).

65. Klonowski, W. Personalized neurological diagnostics from biomedical physicist's point of view and application of new non-linear dynamics methods in biosignal analysis. International journal of biology and biomedical engineering 5, 190-200 (2011).

66. Gomes, R. L., Vanderlei, L. C. M., Garner, D. M., Vanderlei, F. M. \& Valenti, V. E. Higuchi fractal analysis of heart rate variability is sensitive during recovery from exercise in physically active men. MedicalExpress 4 (2017)

67. Martinez-Lavin, M., Infante, O. \& Lerma, C. Hypothesis: the chaos and complexity theory may help our understanding of fibromyalgia and similar maladies. Seminars in arthritis and rheumatism 37, 260-264, https://doi.org/10.1016/j. semarthrit.2007.04.003 (2008).

68. Malliani, A. Principles of cardiovascular neural regulation in health and disease. Vol. 6 (Springer Science \& Business Media, 2000).

69. Faes, L., Zhao, H., Chon, K. H. \& Nollo, G. Time-varying surrogate data to assess nonlinearity in nonstationary time series: application to heart rate variability. IEEE transactions on biomedical engineering 56, 685-695 (2008).

70. Kanters, J. K., Holstein-Rathlou, N. H. \& Agner, E. Lack of evidence for low-dimensional chaos in heart rate variability. Journal of cardiovascular electrophysiology 5, 591-601 (1994).

71. Porta, A. et al. Complexity and nonlinearity in short-term heart period variability: comparison of methods based on local nonlinear prediction. IEEE Transactions on Biomedical Engineering 54, 94-106 (2006).

72. González, J. J., Cordero, J. J., Feria, M. \& Pereda, E. Detection and sources of nonlinearity in the variability of cardiac RR intervals and blood pressure in rats. American Journal of Physiology-Heart and Circulatory Physiology 279, H3040-H3046 (2000).

73. Braun, C. et al. Demonstration of nonlinear components in heart rate variability of healthy persons. American Journal of PhysiologyHeart and Circulatory Physiology 275, H1577-H1584 (1998).

\section{Acknowledgements}

We acknowledge the Extremadura Association of Fibromyalgia (AFIBROEX) in Cáceres (Spain) for helping to recruit the participants for this study. This study was co-funded by the Spanish Ministry of Economy and Competitiveness (reference no DEP2015-70356) in the framework of the Spanish National R+D + i Plan. This study was also funded by the Research Grant for Groups (GR18155 and GR18129) funded by Junta de Extremadura (Regional Government of Extremadura) and European Regional Development Fund (ERDF/ FEDER) 'a way of doing Europe'. The author SV was supported by a grant from the regional department of economy and infrastructure of the Government of Extremadura and the European Social Fund (PD16008). The funders played no role in the study design, the data collection and analysis, the decision to publish, or the preparation of the manuscript.

\section{Author contributions}

S.V., N.G. and D.C.M. conceived and designed research. S.V. and J.P.F.G. conducted experiments. N.G. and F.J.D.M. contributed new reagents or analytical tools. F.J.D.M., J.P.F.G. and S.V. analyzed data. D.C.M., S.V. and N.G. wrote the manuscript. All authors read and approved the manuscript.

\section{Competing interests}

The authors declare no competing interests.

\section{Additional information}

Correspondence and requests for materials should be addressed to D.C.-M.

Reprints and permissions information is available at www.nature.com/reprints.

Publisher's note Springer Nature remains neutral with regard to jurisdictional claims in published maps and institutional affiliations.

Open Access This article is licensed under a Creative Commons Attribution 4.0 International License, which permits use, sharing, adaptation, distribution and reproduction in any medium or format, as long as you give appropriate credit to the original author(s) and the source, provide a link to the Creative Commons license, and indicate if changes were made. The images or other third party material in this article are included in the article's Creative Commons license, unless indicated otherwise in a credit line to the material. If material is not included in the article's Creative Commons license and your intended use is not permitted by statutory regulation or exceeds the permitted use, you will need to obtain permission directly from the copyright holder. To view a copy of this license, visit http://creativecommons.org/licenses/by/4.0/.

(C) The Author(s) 2020 